\title{
Efficient genome engineering of Toxoplasma gondii using the TALEN technique
}

\author{
Hongmei Chen ${ }^{1 \dagger}$, Yijia Guo ${ }^{1 \dagger}$, Yushu Qiu' ${ }^{1}$ Huanbin Huang ${ }^{1}$, Changqing Lin ${ }^{1}$, Min Liu ${ }^{1}$, Xiaoguang Chen ${ }^{1}$, \\ Peiliang Yang ${ }^{2^{*}}$ and Kun Wu${ }^{1 *}$
}

\begin{abstract}
Background: Aromatic amino acid hydroxylase $2(\mathrm{AAH} 2)$ is a bradyzoite-specific upregulated protein that may alter host behaviour by altering the host dopaminergic pathway. To better understand the role of the parasite's AAH2 in host-parasite interactions, we generated an AAH2 fluorescent marker strain of T. gondii using the TALEN technique.

Methods: We generated an AAH2 fluorescent marker strain of T. gondii, which was designated PRU/AAH2-eGFP, using the TALEN technique. This strain stably expressed pyrimethamine resistance for screening and expressed enhanced green fluorescent protein (eGFP)-tagged AAH2 in the bradyzoite stage. The bradyzoite conversion of PRU/ AAH2-eGFP was observed both in vitro and in vivo. The fluorescence localization of AAH2 in mouse models of chronic infection was observed by a Bruker in vivo imaging system.
\end{abstract}

Results: Transgenic T. gondii was successfully generated by the TALEN system. The eGFP-tagged AAH2 could be detected by in vivo imaging.

Conclusions: This study verified the feasibility of using TALEN technology for T. gondii research and provided an in vivo imaging method for in vivo research of bradyzoite-stage proteins.

Keywords: TALEN, Toxoplasma gondii, Bradyzoite, Tyrosine hydroxylase 2, In vivo imaging

\section{Background}

Toxoplasma gondii is an obligate intracellular protozoan parasite and one of the most widespread zoonotic parasites, and it can infect most warm-blooded animals and humans $[1,2]$. This species infects up to a third of the world's population $[3,4]$ and represents a serious threat to public health. Most healthy adults usually show symptoms of latent infection after infection with $T$. gondii. At this time, the parasites convert to bradyzoites and exist in the form of cysts in the brain and muscle tissue of the host [5], which causes latent infection and may lead to changes in several functions of the host brain and behaviour [6]. As models for human infections, rodents

\footnotetext{
*Correspondence: plyang@smu.edu.cn; wksmu@163.com

${ }^{\dagger}$ Hongmei Chen and Yijia Guo contributed equally to this work

1 Department of Pathogen Biology, Guangdong Provincial Key Laboratory

of Tropical Disease Research, School of Public Health, Southern Medical

University, Guangzhou 510515, China

${ }^{2}$ Experimental Animal Center, Nanfang Hospital, Southern Medical

University, Guangzhou 510515, China
}

have been studied extensively as intermediate hosts of $T$. gondii because these animals are commonly preyed upon by felids, which are the only definitive hosts of this parasite [7]. According to behavioural studies, mice with chronic T. gondii infection exhibit significant changes in reactions, spatial learning, locomotion, memory and the ability to learn new things $[6,8,9]$. These effects are considered to be a result of manipulation by the parasite to increase mouse susceptibility to predation, which leads to successful transmission of the parasite to the feline host $[10,11]$. The bradyzoite stage shows a preference for the brain of its intermediate host, which supports the role of $T$. gondii in host manipulation [12].

Studies have shown that $T$. gondii may alter host behaviour by altering the host dopaminergic pathway and increasing dopamine levels in the brain [13-15]. Tyrosine hydroxylase $(\mathrm{TH})$, a member of the aromatic amino acid hydroxylase (AaaH) family, is widespread in insects, mammals and humans and represents the rate-limiting enzyme in the synthesis of dopamine. The genome of $T$. 
gondii was found to contain two AaaH-coding sequences, namely AAH1 and AAH2, which encode tyrosine hydroxylases with signal peptides [16]. Studies have shown that AaaHs play an important role in the function of the brain, and the genes encoding these enzymes are among the likely candidates for genes associated with schizophrenia [17]. Meanwhile, latent $T$. gondii infection is one of the factors leading to schizophrenia, and serological surveys of $T$. gondii infection have shown that there is a positive correlation between the rate of seropositivity and incidence of schizophrenia [18, 19]. In contrast with AAH1, which is constitutively expressed in tachyzoites and bradyzoites, AAH2 is specifically upregulated in bradyzoite cysts [16], which is the form of the parasite that is present during chronic infection. Due to its bradyzoite-specific upregulation of expression and unique predicted signal peptide, it is particularly important to further characterize the localization and function of AAH2.

Gene function research is closely associated with gene editing technology. For a long time, transgenic T. gondii was constructed by transfecting cells with donor DNA containing a long homologous sequence. However, homologous recombination (HR) occurs at a very low frequency; therefore, the screening and isolation of parasites was time consuming. Newly developed customdesigned nucleases, namely zinc finger nucleases (ZFNs), transcription activator-like effector nucleases (TALENs) and the clustered regularly interspaced short palindromic repeat (CRISPR)/CRISPR-associated (Cas) system, have shown high editing efficiency. However, the assembly of functional ZF proteins with the desired DNA binding specificity is laborious and time consuming because it requires an extensive screening process. Moreover, ZF domains exhibit context-dependent binding preference due to crosstalk between adjacent modules when assembled into a larger array [20]. The discovery of transcriptional activator-like effectors (TALEs) from Xanthomonas bacteria [21-25] was a breakthrough that simplified the generation of custom TALE DNA-binding domains with programmable specificity [26, 27]. Similar to ZFNs, a pair of TALENs can be designed to induce a targeted double-strand break (DSB) at the desired chromosomal locus, which is repaired by HR when provided with an exogenous donor plasmid containing homologous sequences flanking the cut site. DNA DSBs generated by targeted nucleases dramatically stimulate $\mathrm{HR}$ $[27,28]$, and TALEN technology has been successfully applied in several species. However, the accessibility of this technology in $T$. gondii has not yet been reported. Recently, due to the convenience and high efficiency of multiplex genome editing, CRISPR/CAS9 has proven to be useful for several types of genome modifications in model organisms, including T. gondii [29, 30]. However, in contrast with ZFNs and TALENs, the CRISPR/CAS9 system can tolerate small mismatches, insertions and other mutations in the target sequence, which leads to increased off-target effects [31, 32].

Herein, we chose the T. gondii PRU strain [33] to construct an AAH2 fluorescent marker strain using the TALEN technique. The tachyzoite-bradyzoite interconversion of transgenic T. gondii was triggered in vitro by using high-pH medium and in vivo by establishing chronically infected mouse models. The fluorescence of eGFP-tagged AAH2 protein in the brain tissue of mice during the latent phase could be observed by an in vivo imaging system, allowing determination of the localization of AAH2 in the animal model and confirming the feasibility of TALEN technology for the study of $T$. gondii gene function.

\section{Results}

Identification of TALENs plasmids and donor plasmid

The TALENs and homology template were designed to target and tag the AAH2 gene with eGFP to generate transgenic $T$. gondii. Recombinant plasmids were verified by PCR and the double-enzyme cleavage method. TALE-L was cloned into pL62, and TALE-R was cloned into pR54. The recombinant plasmids PTALEN-L and pTALEN-R were verified by the double-enzyme cleavage method with BamHI and PstI.

The SAG1 gene promoter was amplified by PCR and inserted into pTALEN-L digested with AscI/SpeI. The recombinant plasmid pTALEN-L-SAG1 was digested with AscI/SpeI to indicate successful insertion. The GRA2 gene terminator was amplified via PCR and inserted into pTALEN-L-SAG1 digested with BglII/NotI. The recombinant plasmid pTALEN-L-SG was digested with BglII/Not I to indicate successful insertion (Additional file 1: Figure S1a, b).

The GRA2 gene terminator sequence was amplified via PCR and inserted into pTALEN-R digested with ApaI/NotI. The recombinant plasmid pTALEN-R-GRA2 was digested with $B g l \mathrm{II} / \mathrm{Not} \mathrm{I}$ to indicate successful insertion. The DHFR* and SAG1 gene promoters were amplified by PCR and inserted into pTALEN-R-GRA2 digested with AscI/SpeI. The recombinant plasmid pTALEN-RDSG was digested with AscI/SpeI to indicate successful insertion (Additional file 1: Figure S1c, d).

The AAH2 homologous left arm, eGFP gene, DHFR* and $\mathrm{AAH} 2$ homologous right arm were amplified via PCR from the recombinant plasmid pZEDY. The fragment ZEDY was also amplified and inserted into pUC19 digested with $B a m H I$. The donor plasmid pZEDY was digested with BamHI to indicate successful insertion (Additional file 2: Figure S2). 


\section{Verification of transgenic T. gondii PRU/AAH2-eGFP}

To further verify the feasibility of the technology used for editing the AAH2 gene, T. gondii PRU strain tachyzoites were co-transfected with three recombinant plasmids, namely pTALEN-L-SG, pTALEN-R-DSG and the donor plasmid, and transgenic parasites designed to express fluorescence-tagged AAH2 protein in the bradyzoite stage were identified as follows. The transgenic parasites were screened with pyrimethamine and confirmed by PCR using gDNA as template. The identification PCR primers were designed. PCR1 and PCR2 each had one primer that was not bound on the homologous arm but rather on the toxoplasma genome sequence upstream and downstream of the homologous arms. The fragments amplified by PCR1 and PCR2 both contained part of the inserted genes (eGFP and DHFR*), homologous arms and some genomic sequence beyond the homologous arms (Fig. 1a). A verification PCR assay demonstrated that the eGFP and DHFR* genes were inserted accurately by the TALEN technology (Fig. 1b).

\section{Expression of AAH2-eGFP in bradyzoites induced in vitro}

To express the bradyzoite-specific protein AAH2, a tachyzoite-bradyzoite conversion system of transgenic T. gondii was established in vitro. The status of the parasites and the expression of fluorescence were monitored by inverted fluorescence microscopy. On the first to third days, the parasites invaded the host cell and began to reproduce, and the parasitophorous vacuole could be observed on the third day. The growth and reproduction of the test group was slower than that of the control group (Fig. 2a1-a3, c1-c3), and no specific fluorescence was observed for the first 3 days (Fig. 2b1-b3, d1-d3).
On the fourth and fifth days, cyst-like structures could be observed in the test group (Fig. 2c4, c5). Specific fluorescence was not observed in the transgenic bradyzoites induced in vitro (Fig. 2b4, b5, d4, d5). However, tachyzoite-bradyzoite conversion in vitro cannot fully reflect the expression of bradyzoite-stage proteins in vivo.

RNA from the parasites in the control and test groups was extracted to establish a cDNA library for further identification. Bradyzoites induced in vitro in the test group were verified by RT-PCR using the bradyzoite-specific BAG1 primers P27 and P28. The tachyzoite-specific SAG1 was amplified from cDNA using the primers P29 and P30 as a control. The verification PCR assay showed that the in vitro induction of transgenic T. gondii bradyzoites was successful (Fig. 3). The expression of eGFP and DHFR* was identified by RT-PCR using the primers P31/P32 and P33/P34. Verification PCR showed that the resistance gene was expressed at both the tachyzoite stage and the in vitro-induced bradyzoite stage, as expected; however, the expression of eGFP was not detected in the test group (Fig. 3).

\section{Expression of AAH2-eGFP in bradyzoites induced in vivo}

To observe the fluorescence expression of the transgenic T. gondii cysts in the host, a chronic infection animal model of transgenic T. gondii was established by infecting Kunming mice with PRU/AAH2-eGFP. In contrast with the brains of PRU/WT tachyzoite-infected mice, the brains of the PRU/AAH2-eGFP-infected mice exhibited a bradyzoite-specific fluorescence-tagged AAH2 signal within cysts, showing that bradyzoites of transgenic $T$. gondii had formed in vivo and that a specific fluorescent

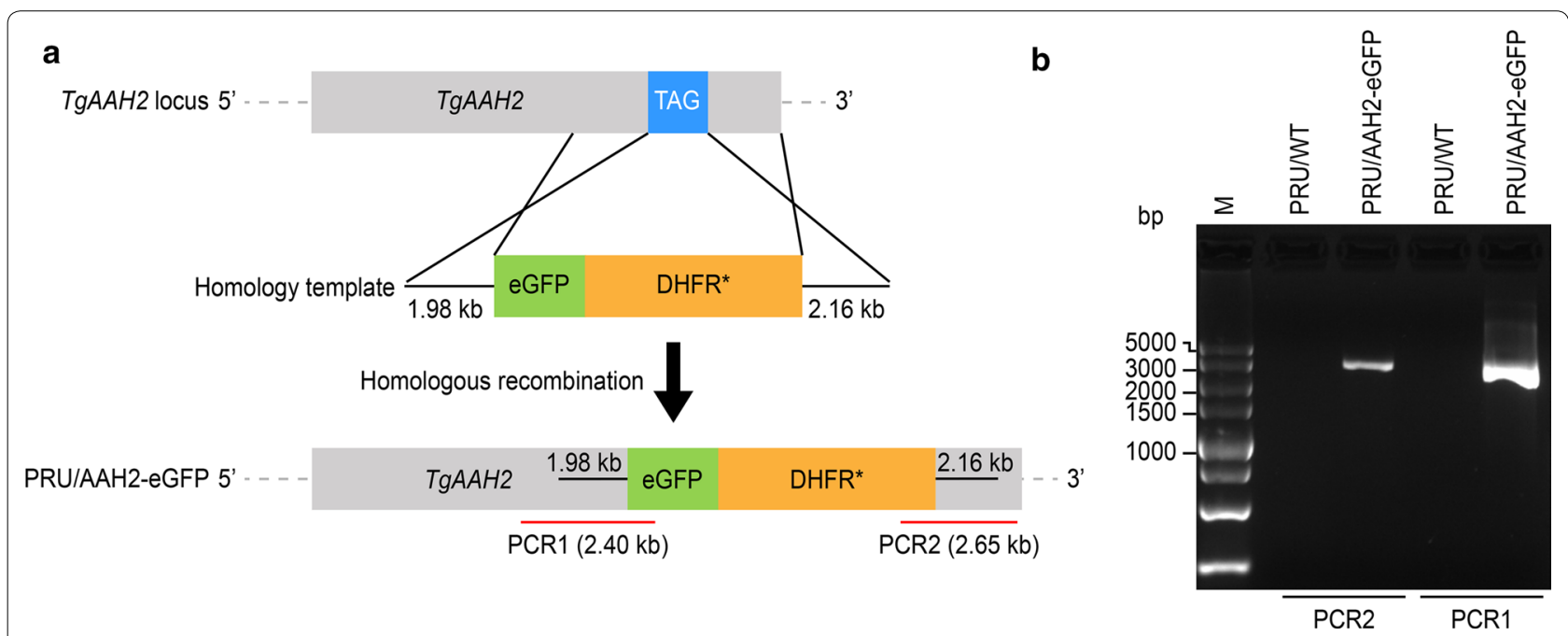

Fig. 1 TALEN system tagging of the AAH2 locus. a Schematic illustration of the TALEN strategy used to tag AAH2 at the C-terminus with eGFP. $\mathbf{b}$ Verification PCR showing the correct insertion of eGFP and DHFR* 


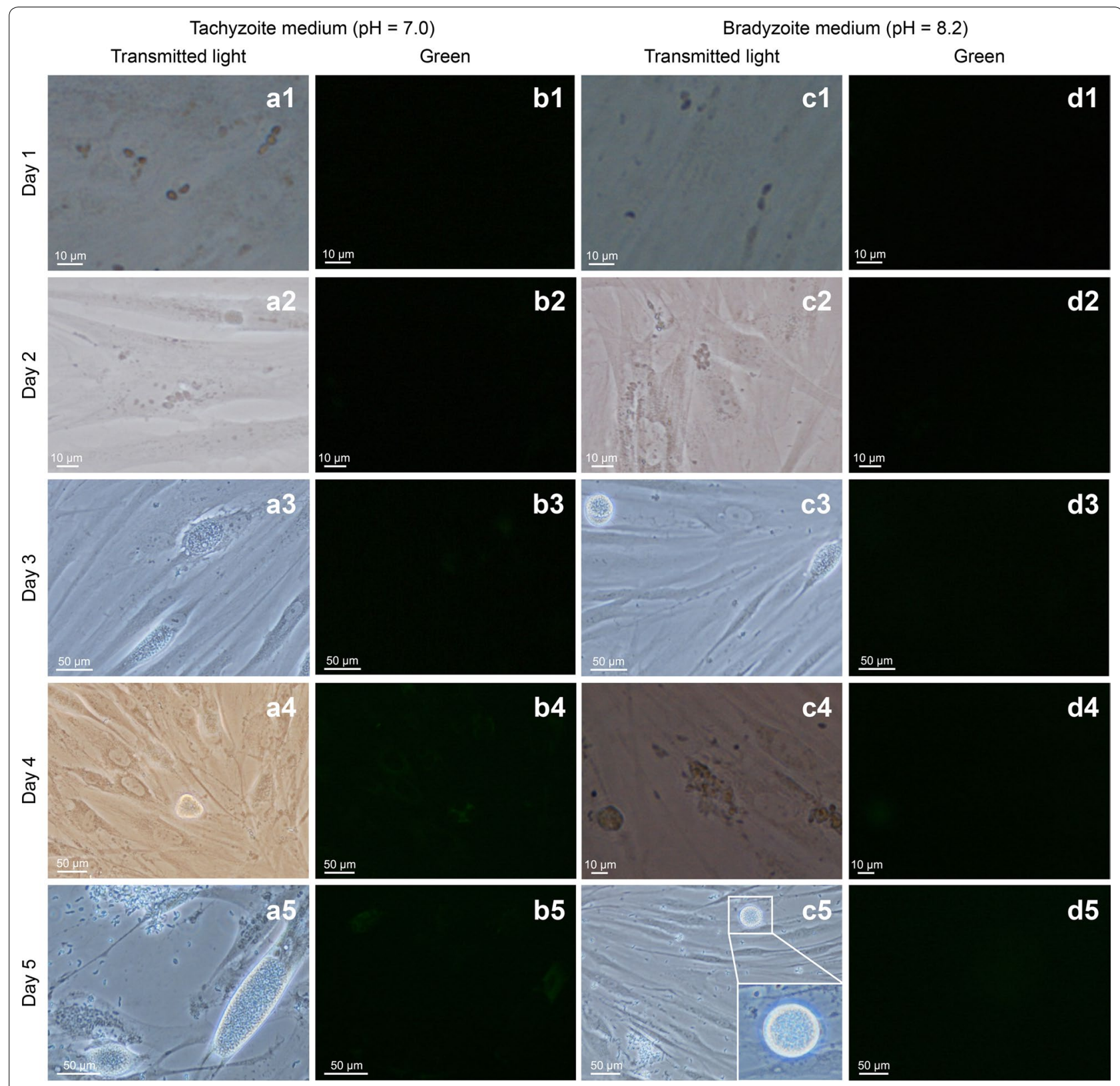

Fig. 2 In vitro growth of tachyzoite-bradyzoite conversion and fluorescence microscopy analysis of transgenic T. gondii. HFF cells were infected with PRU/AAH2-eGFP tachyzoites and cultured in medium at pH 7.0 (a, b) or pH $8.2(\mathbf{c}, \mathbf{d})$. Transmitted light images $(\mathbf{a}, \mathbf{c})$ and green fluorescence images (b, d) were obtained by fluorescence microscopy. Scale-bars: $10 \mu \mathrm{m}, 50 \mu \mathrm{m}$

signal for the transgenic T. gondii cysts could be observed (Fig. 4).

RT-PCR was performed to further identify the status of the transgenic T. gondii PRU/AAH2-eGFP during the chronic phase. Amplification of the fragment of the BAG1 gene from infected brain tissue using primers P27 and P28 indicated that the in vivo induction of transgenic $T$. gondii bradyzoites was successful and that animal models of transgenic $T$. gondii chronic infection had been established. The positive fragments of the DHFR* gene were amplified by PCR from transgenic $T$. gondii gDNA, infected brain tissue cDNA and transgenic $T$. gondii tachyzoite cDNA using primers P33 and P34, and the results indicated that the resistance gene was stably expressed at both the tachyzoite and bradyzoite stages. Fragments of the AAH2-eGFP gene were only amplified via PCR using primers P35 and P36 from transgenic $T$. gondii gDNA and infected brain tissue cDNA and not 


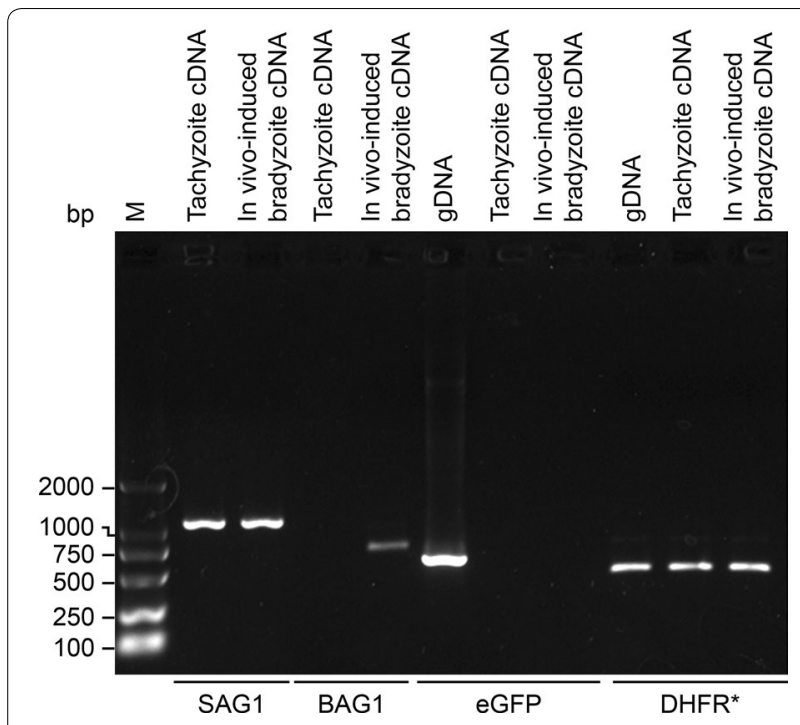

Fig. 3 Identification of in vitro-induced bradyzoites and expression of inserted genes in transgenic T. gondii. RT-PCR verification of the tachyzoite-specific protein SAG1 and bradyzoite-specific protein BAG1 in PRU/AAH2-eGFP tachyzoites and in vitro-induced bradyzoites, respectively. RT-PCR verification of the expression of the inserted genes eGFP and DHFR* compared with the PCR verification of the inserted genes from transgenic T. gondii gDNA from the cDNA of transgenic T. gondii tachyzoites, which showed that the bradyzoite-specific eGFP-tagged AAH2 gene had been transcribed and could be detected only at the bradyzoite stage as expected (Fig. 5).

The fluorescence images and verification PCR assay demonstrated that the transgenic T. gondii strain PRU/ AAH2-eGFP was successfully generated by the TALEN technology and the inserted fluorescence gene and pyrimidine resistance gene were successfully expressed in this experiment.

\section{Discussion}

\section{Amino acid hydroxylases from the bradyzoite stage of $T$. gondii}

Acute infection with $T$. gondii can lead to teratogenesis, miscarriage and even death from toxoplasmic encephalitis in immunocompromised individuals; therefore, previous studies have mainly focused on the tachyzoite stage of T. gondii and the immune interaction between tachyzoite and the host, and the bradyzoite has been considered to have little effect on the host. Therefore, limited research has been performed on the interaction between bradyzoite-specific proteins and the host. However, in recent years, a growing number of studies have shown that $T$. gondii cysts caused by chronic infection

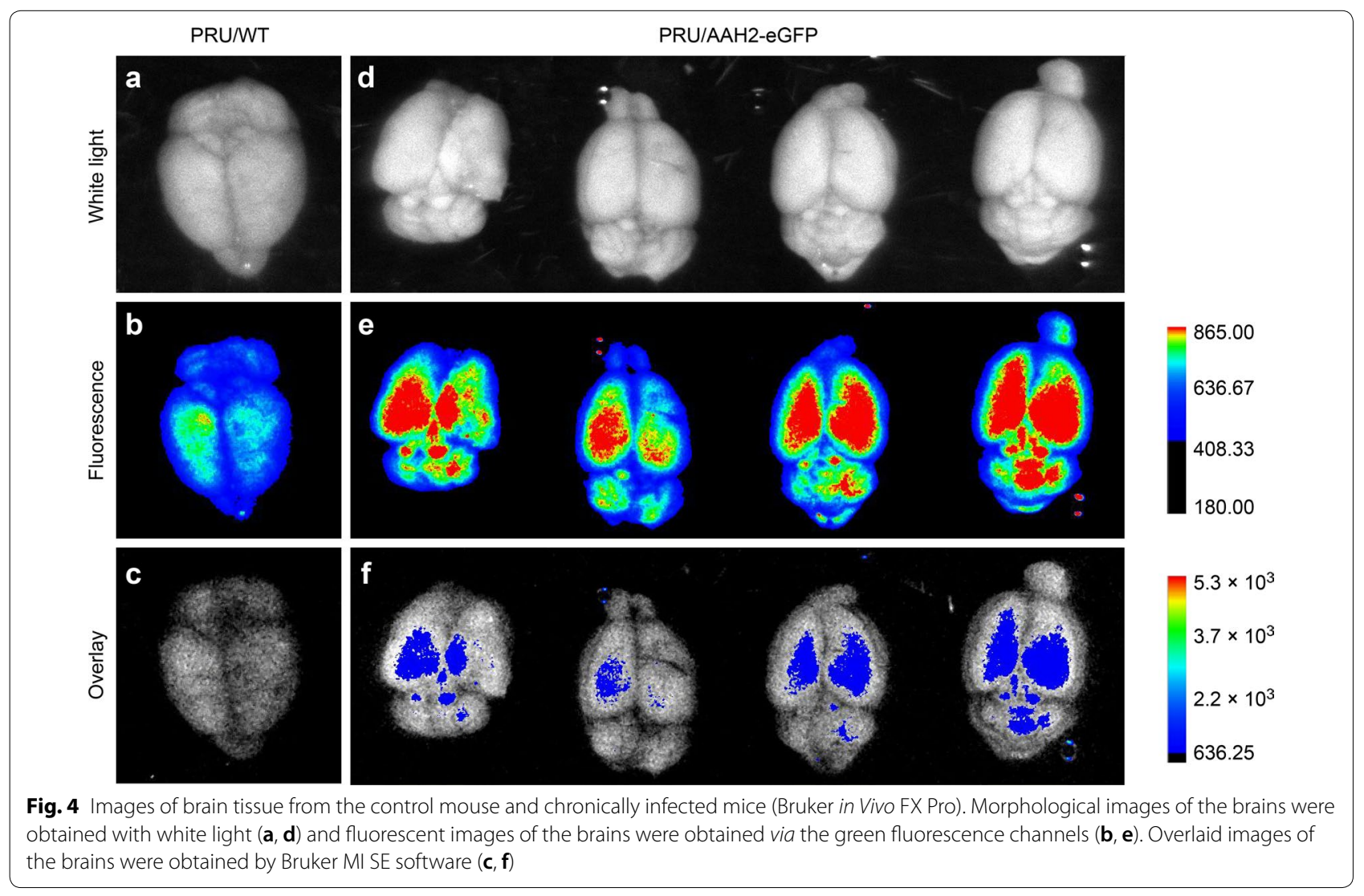




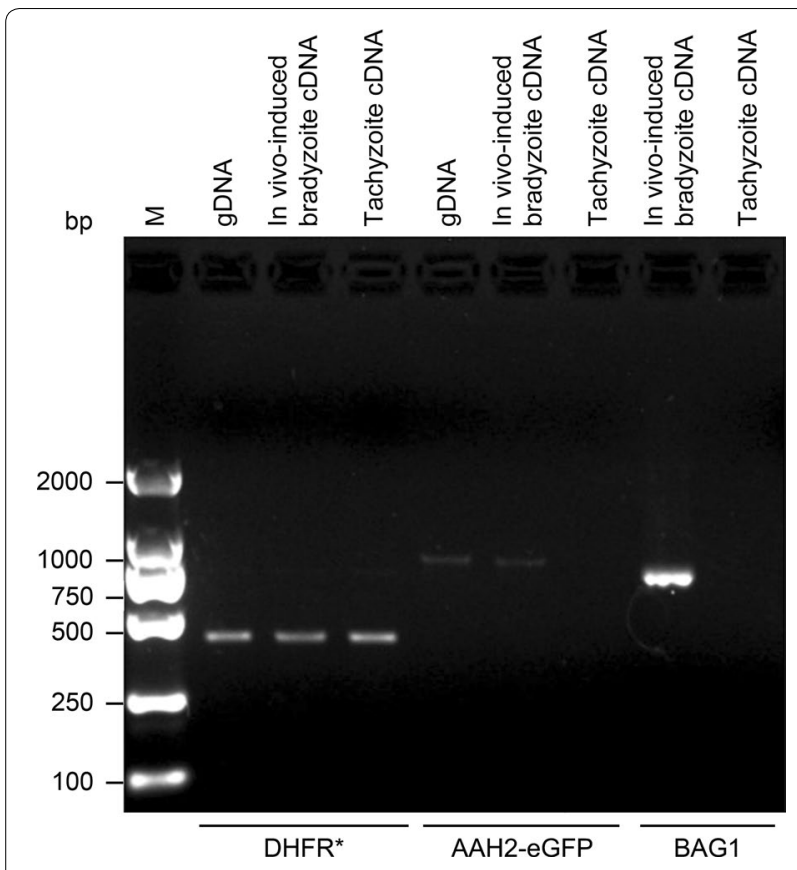

Fig. 5 Identification of transgenic T. gondii cysts and expression of inserted genes in infected brain tissue. PCR verification of inserted DHFR* from different templates of PRU/AAH2-eGFP. PCR verification of AAH2-eGFP from different templates of PRU/AAH2-eGFP. RT-PCR verification of bradyzoite-specific BAG1 in PRU/AAH2-eGFP-infected brain tissue indicated the formation of cysts

(bradyzoite stage) are correlated with impairment of the function of the host brain and cognitive ability due to neurotropism. Therefore, our team focused on the study of bradyzoite-specific proteins and aimed to explore the interaction between the bradyzoites in cysts and neurotransmitters of the host.

Amino acid hydroxylases from $T$. gondii have received much attention in recent years in the context of interactions between parasites and hosts. Interestingly, the two amino acid hydroxylases in T. gondii mentioned previously share $98 \%$ sequence similarity with each other [16] and are homologous to phenylalanine hydroxylase. Attempts to delete AAH1 using the efficient CRISPRCas9 method have been unsuccessful to date [34] and AAH1 may be essential for the growth and development of parasites. Deletion of AAH2 did not affect tachyzoite or bradyzoite growth in tyrosine-rich or tyrosine-limiting medium [35], and the role of AAH2 in either stage of the life-cycle remains unidentified [13, 34]. Furthermore, recent studies have demonstrated that parasitesecreted AAH is not necessary for the generation of risky behaviour or the increased trappability observed during chronic T. gondii infection [36], and AAH2 is not required for tyrosine metabolism in these two developmental forms [35]. Recent studies have found that AAH2 is not required for the DA-dependent neurobehavioral abnormalities caused by $T$. gondii infection [37], but this subject remains controversial. Why is AAH2 expressed at a low level in tachyzoites and upregulated in bradyzoites [16]? This question is of great interest to researchers, which is why AAH2 was the first gene that we studied. In the course of this study, we established a method for editing targeted genes. Because the location of AAH2 in the brain is likely to be a critical factor for its effect on host behaviour, identifying this location during infection is crucial. Here, we generated an eGFP-tagged AAH2 $T$. gondii strain via TALEN technology to further characterize the localization of AAH2 in the bradyzoite stage.

\section{Genome editing technologies in T. gondii}

In life science, research on gene functions is the basis for exploring pathogenic mechanisms, and advances in genome editing technologies have led to breakthroughs in the study of gene function. Artificial nucleases developed in recent years are tools that can conveniently and accurately edit genomes of interest. Currently, three main types of nucleases have been successfully used for genome editing: ZFNs, TALENs and CRISPR systems. CRISPR/CAS9 with a pyrimidine-resistant screening system is now widely used for genome editing of $T$. gondii, although the off-target effects of this system are caused by the recognition of target DNA by the single guide RNA (sgRNA) based on only $20 \mathrm{nt}$, and this drawback needs to be overcome. In contrast with the sgRNA of CRISPR/CAS9, the target DNA is recognized by repeat domains using TALEN technology. Each domain contains 33-35 amino acids, and one repeat domain corresponds to $1 \mathrm{nt}$, which guarantees the specificity of the cleavage site and leads to reduced off-target effects [32]. Here, we selected TALENs, a tool with reduced off-target effects, to develop an additional method to edit the T. gondii genome. In this study, the TALEN system was used to generate a transgenic T. gondii PRU strain, which was designated PRU/AAH2-eGFP. This strain could stably express an eGFP-tagged AAH2 fusion protein and was identified by verification PCR. The results demonstrate the feasibility of using TALEN technology for gene function research of $T$. gondii and provide a molecular technical basis for the mechanism of action of the T. gondii tyrosine hydroxylase in the host.

Although TALEN technology has high specificity and low off-target effects, it is complicated and time consuming to reconstruct TALEN plasmids to function normally in T. gondii. Using a FastTALE TALEN Assembly Kit, we achieved rapid assembly of TALEs, which recognize the target sequence. SAG1 is a $30-\mathrm{kDa}$ surface protein of $T$. gondii tachyzoites that represents 3 to $5 \%$ of the total proteins in this parasite [33], and Soldati \& Boothroyd [38] 
have successfully produced an RFP expression system with a SAG1 gene promoter. In this study, we recombined the TALEN plasmids using the SAG1 gene promoter to replace the CMV promoter for the expression of TALENs in T. gondii for gene editing. Transfection of recombinant plasmids containing the DHFR* mutation in individual bases into WT strains of T. gondii and the use of pyrimethamine to screen transgenic parasites have been widely used in transgenic technology [29, 30, 38, 39]. In the process of constructing the TALEN system, including the TALEN plasmids and donor plasmids as homologous templates, we also used a set of convenient methods for constructing recombinant plasmids, such as SOE PCR and In-Fusion technology.

\section{In vitro and in vivo studies of bradyzoite stage proteins}

Due to the bradyzoite-specific expression of AAH2, the high-pH shock method was applied to induce tachyzoitebradyzoite conversion of transgenic parasites in vitro. Upon successful bradyzoite induction of transgenic parasites, which was verified by RT-PCR of the bradyzoitespecific BAG1 gene, we observed that the reproduction of tachyzoites was distinctly retarded and that cyst-like structures could be observed under the conditions of the inducible medium. We hypothesized that the reproduction may have been changed by the conditions in the culture medium as well as by the conversion to the bradyzoite stage, which may have been induced simultaneously. However, no fluorescence was observed in vitro, and the transcriptional expression of AAH2-eGFP was not detected. The reasons underlying this observation may be as follows. First, expression of the BAG1 protein begins in the early stage of bradyzoites [40], and the AAH2 protein may not be expressed during short-term induction in vitro. Secondly, tachyzoite-bradyzoite conversion in vitro does not fully reflect the conditions of the cyst in the host and may affect the expression of the bradyzoite-specific gene.

To explore the condition of bradyzoites in the cyst in host, chronic infection models were established in this study to further analyse the expression of the AAH2 protein in the host. In contrast with those of the control mice, fluorescence images and verification PCR of mice infected by transgenic parasites demonstrated that the inserted fluorescence gene and pyrimidine resistance gene were successfully expressed. The successful construction of an AAH2 fluorescent marker strain PRU/ AAH2-eGFP allowed us to further study the secretion process of AAH2 protein. Studies have found that AAH2 has a signal sequence motif, suggesting that the protein is a secreted protein. Using PRU/AAH2-eGFP, we can further explore whether the AAH2 protein can be secreted outside the cysts during the bradyzoite stage and interact with the host brain tissue, which will provide useful clues for further study of the regulatory role of tyrosine hydroxylase of $T$. gondii in the host dopamine metabolic pathway. Researchers are interested in tachyzoite-bradyzoite interconversion and have established an in vitro model of parasite interconversion in host cells; however, few in vivo studies have been performed on the interaction of bradyzoite-specific proteins with the host nervous system. The results of this study indicate that studies of bradyzoite-stage proteins are best conducted in host models in vivo because the interconversion of parasites in the host is closely associated with host immunity. The present study provides a convenient and quick method to examine the fluorescence localization of bradyzoite-specific proteins, namely in vivo imaging. In vivo research is the ultimate tool for the investigation of biological reactions, and direct imaging results can provide clear information at a glance.

\section{Conclusions}

The generation of PRU/eGFP-AAH2 indicates that TALENs can specifically target and successfully edit specific genes in the $T$. gondii genome, thus providing a strategy for exploring $T$. gondii gene function. However, certain limitations were observed in our study, and further research is required for the construction of fluorescencetagged AAH2 to be used to trace the secretory pathway of AAH2 in parasites and further study the interactions of the parasite with the host nervous system. A transgenic T. gondii that can stably express fused bradyzoite stagespecific fluorescent protein can be used to determine the localization of AAH2, which may indicate the role of the parasite's AAH2 in host-parasite interactions and could contribute to further understanding the upregulation of AAH2 in bradyzoites.

\section{Methods \\ Mice}

Female 6-week-old Kunming mice were purchased from the Animal Experimental Centre of Southern Medical University (Guangzhou, China). The Kunming mice were infected with parasites, and their brain tissue was excised after euthanasia by cervical dislocation. Fluorescence localization of the AAH2 protein in the brain tissue was evaluated using an in vivo imaging system (Bruker FX Pro, Billerica, MA. USA).

\section{Cells and parasite cultures}

The PRU strain of $T$. gondii was maintained in human foreskin fibroblasts (HFFs) cultured in tachyzoite medium [Dulbecco's modified Eagle's medium (DMEM; Gibco, Beijing, China), 1\% foetal bovine serum (FBS; Gibco, Newcastle, Australia), 1\% penicillin and 
streptomycin (Gibco, Frederick, MD.USA)] at $37{ }^{\circ} \mathrm{C}$ in a $5 \% \mathrm{CO}_{2}$ incubator. All PRU strain parasites were maintained until the parasites lysed $80-100 \%$ of the host cells. For the collection of tachyzoites, the parasites were syringed with a 27 -gauge needle, filtered through a $3.0-\mu \mathrm{m}$-pore filter and then pelleted at $2500 \times \mathrm{rpm}$ for 10 $\min$.

\section{Construction of TALEN plasmids and homology template}

The primer sequences and plasmid structures used in this study are listed in the additional files (Additional file 3: Table S1 and Additional file 4: Table S2). The sequences for primer design and targeting plasmid validation were obtained from ToxoDB (www.toxodb.org) [41].

The TALENs were constructed with the FastTALE TALEN Assembly Kit (Sidansai Biotechnology Co., Shanghai, China). The steps were as follows. According to the sequencing result for the $T$. gondii PRU strain tyrosine hydroxylase type2 (AAH2, locus on chromosome V, annotated as TGME49_212740), we first selected a pair of suitable DNA sequences for gene targeting and named the pair TALE-L/R (Additional file 5: Figure S3). We assembled TALEN modules that recognized one or two bases (Sidansai Biotechnology Co.) into a suitable skeleton vector ( $\mathrm{pL62}$ for L, pR54 for R) that contained CMV promoter and Fork I sequence and constructed pTALEN$\mathrm{L}$ and pTALEN-R using a FastTALE TALEN Assembly Kit according to the manufacturer's protocol (Additional file 6: Figure S4a, b).

To recombine pTALEN-L, the SAG1 gene promoter region (SAG1p-L) was amplified by PCR from genomic DNA (gDNA) isolated from T. gondii PRU strain tachyzoites (DNeasy Blood \& Tissue Kit; Qiagen, Hilden, Germany) cultured in vitro as previously described [42] using primers $\mathrm{P} 1$ and $\mathrm{P} 2$. The terminal region of the GRA2 gene (GRA2t-L) was amplified by PCR from gDNA using primers P3 and P4. The amplified SAG1p-L fragment was digested with the same enzymes used to digest pTALENL, namely AscI and SpeI-HF, and the resulting plasmid was designated pTALEN-L-SAG1. The amplified GRA2t$L$ fragment was digested with the same enzymes used to digest pTALEN-L-SAG1, namely BglII and NotI-HF, and the resulting plasmid was designated pTALEN-L-SAG1GRA2 (pTALEN-L-SG) (Additional file 6: Figure S4c).

To recombine pTALEN-R, the SAG1 gene promoter region (SAG1p-R) was amplified by PCR from gDNA using primers $\mathrm{P} 5$ and $\mathrm{P} 6$. The terminal region of the GRA2 gene (GRA2t-R) was amplified by PCR from gDNA using primers P7 and P8. In addition, the pyrimethamine-resistant DHFR (DHFR*) was amplified from the pLic $3 \times$ HA-DHFR-TS plasmid using primers P9 and P10. The amplified GRA2t-R fragment was digested with the same enzymes used to digest pTALEN-L-GRA2, namely BglII and NotI-HF, and the resulting plasmid was designated pTALEN-R-GRA2. The amplified SAG1p$\mathrm{R}$ fragment and DHFR* were digested with the same enzymes used to digest pTALEN-R-GRA2, namely AscI and SpeI, and the resulting plasmid was designated pTALEN-R-DHFR-TS-SAG1-GRA2 (pTALEN-R-DSG) (Additional file 6: Figure S4d).

To construct a donor plasmid as a homology template, the left arm (Z) was amplified from PRU strain gDNA as a homologous fragment using primers P11 and P12. The right arms (Y1, Y2) of the AAH2 gene were amplified from PRU strain gDNA as homologous fragments using primers P13/P14 and P15/P16. The eGFP gene (E) was amplified from the pR52 plasmid using primers P17 and P18. DHFR* (D) was amplified from pLic $3 \times$ HADHFR-TS using primers P19 and P20. These amplified fragments $\mathrm{Z}, \mathrm{E}, \mathrm{D}, \mathrm{Y} 1$, and $\mathrm{Y} 2$ were extended with the spliced-overlapping-extension (SOE) method and then amplified by PCR (the SOE PCR details are described in Additional file 7: Table S3). The SOE PCR product ZEDY was inserted into the plasmid pUC19 (TaKaRa, Dalian, China), which was linearized by digestion with $\mathrm{BamHI}$ using the In-Fusion enzyme (Clontech, Mountain View, CA. USA), and the resulting donor plasmid was designated pZEDY (Additional file 8: Figure S5).

\section{Generation of the transgenic T. gondii PRU strain}

To generate transgenic parasites expressing eGFP-tagged AAH2 during the bradyzoite stage, electroporation of tachyzoites was performed as previously described [43]. Toxoplasma gondii PRU strain tachyzoites were purified [42] and $4 \times 10^{7}$ tachyzoites were resuspended with $24 \mu \mathrm{g}$ of donor plasmid and approximately $63 \mu \mathrm{g}$ of TALEN plasmid $(21 \mu \mathrm{g}$ of pTALEN-L-SG and $42 \mu \mathrm{g}$ of pTALENR-DSG) in $400 \mu \mathrm{l}$ of cytomix buffer $[120 \mathrm{mM} \mathrm{KCl}, 0.15$ $\mathrm{mM} \mathrm{CaCl}, 10 \mathrm{mM} \mathrm{K} \mathrm{HPO}_{4}-\mathrm{KH}_{2} \mathrm{PO}_{4}, 25 \mathrm{mM}$ HEPES (pH 7.6), 2 mM EDTA (pH 7.6), 5 mM MgCl, 2 mM ATP and $5 \mathrm{mM} \mathrm{GSH}$ (pH 7.6)] [44]. The parasite suspension was transferred to 0.4-cm-gap cuvettes (Bio-Rad Laboratories, Hercules, CA, USA), and electroporation was performed at $1500 \mathrm{~V}, 25 \Omega$, and $25 \mu \mathrm{F}$ using a Gene Pulser $\mathrm{Xcell}^{\mathrm{TM}}$ electroporation system (Bio-Rad Laboratories). After electroporation, the parasites were incubated for 20 $\mathrm{min}$ at room temperature. Then, the parasites were inoculated into HFFs in $25-\mathrm{cm}^{2}$ T-flasks (Corning, Wujiang, China) and cultured in a conventional environment with drug-free culture medium as mentioned above for $24 \mathrm{~h}$ to allow for the action of TALENs on the target gene and the expression of the pyrimethamine-resistant DHFR. Then, pyrimethamine (1 $\mu \mathrm{M}$; Sigma-Aldrich, Buchs, Switzerland) was added to the medium. Stable transgenic parasites were selected in DMEM supplemented with high concentrations of pyrimethamine $(2-3 \mu \mathrm{M})$ for 
20 generations (2nd to 15 th generation, $2 \mu \mathrm{M}$; 16th and above, $3 \mu \mathrm{M}$ ) [45]. After screening of several generations, the pyrimethamine-resistant parasites were identified by PCR. The confirmed parasites were designated PRU/ AAH2-eGFP, and they could express eGFP-tagged AAH2 during the bradyzoite stage (cyst stage).

\section{Tachyzoite-bradyzoite conversion of PRU/AAH2-eGFP in vitro}

The high-pH shock method [46] was applied to induce the stage conversion of tachyzoites to bradyzoites in vitro. First, $5 \times 10^{4}$ PRU/AAH2-eGFP tachyzoites were inoculated into HFFs in $25-\mathrm{cm}^{2}$ T-flasks and cultured for $4 \mathrm{~h}$ in tachyzoite medium to allow tachyzoites to invade the cells. To establish a tachyzoite-to-bradyzoite interconversion system, the tachyzoite medium was replaced with bradyzoite medium [RPMI 1640 (Gibco, Beijing, China), $1 \mathrm{~g} / 500 \mathrm{ml} \mathrm{NaHCO}$ (Sigma-Aldrich, Saint. Louis, MO. USA), 10 mM HEPES (Sigma-Aldrich, Saint. Louis, MO. USA), 1\% FBS and 1\% penicillin and streptomycin at $\mathrm{pH}$ 8.2] as a test group, and the lid of the flask was sealed to isolate parasites from the $5 \% \mathrm{CO}_{2}$ environment to maintain an alkaline $\mathrm{pH}$. Additionally, the medium was changed daily. Simultaneously, the tachyzoite medium in another flask was replaced with tachyzoite medium as a control. Parasite morphology was observed using an inverted microscope (Nikon, Tokyo, Japan), and the expression of fluorescence was observed daily by inverted fluorescence microscopy (Nikon). After 5 days of induction, two groups of parasites were purified to extract RNA and establish cDNA for identification.

\section{Tachyzoite-bradyzoite conversion of PRU/AAH2-eGFP in vivo}

To establish the chronically infected model, 6-week-old female Kunming mice $(n=4)$ were infected intraperitoneally (i.p.) with PRU/AAH2-eGFP, and one mouse was i.p. infected with PRU/wild-type (WT) tachyzoites as a control. Because the skulls and hair of mice would interfere with imaging, the brains of the PRU/AAH2eGFP-infected mice and PRU/WT-infected mouse were excised to obtain images of fluorescence expression in bradyzoites induced in vivo after 3 months of infection. The brain tissue was kept intact and washed in normal saline to remove blood and stained hair and then placed in a disposable culture dish for photography. Standard exposure and an optimized exposure time were used. A multiwavelength illumination source and an excitation wavelength of $498 \mathrm{~nm}$ and emission wavelength of $516 \mathrm{~nm}$ were selected according to the fluorophore of the sample (eGFP). After the programme was established, the brain samples were directly imaged using a Bruker in vivo imaging system (Bruker FX Pro) to observe the fluorescence localization of the AAH2 protein during the latent phase (cyst stage) in vivo. Then, the brain tissues were immediately ground into homogenate for RT-PCR identification.

\section{Additional files}

Additional file 1: Figure S1. Construction and identification of TALEN plasmids.

Additional file 2: Figure S2. Identification of the recombinant donor plasmid pZEDY.

Additional file 3: Table S1. Nucleotide sequences of all primers designed in this study.

Additional file 4: Table S2. Structures of the plasmids used in this study.

Additional file 5: Figure S3. TAL effector nucleases designed for specific targeting of $\mathrm{TgAAH} 2$.

Additional file 6: Figure S4. Steps for constructing TALEN plasmids.

Additional file 7: Table S3. SOE PCR to extend and amplify ZEDY.

Additional file 8: Figure S5. Schematic of donor plasmid construction.

\section{Abbreviations}

AAH2: aromatic amino acid hydroxylase 2; eGFP: enhanced green fluorescence protein; TH: tyrosine hydroxylase; AaaH: aromatic amino acid hydroxylase; HR: homologous recombination; ZFNs: zinc finger nucleases; TALENs: transcription activator-like effector nucleases; CRISPR/Cas: clustered regularly interspaced palindromic repeats/CRISPR-associated proteins; TALEs: transcription activatorlike effector nucleases; DSB: double-strand break; HFFs: human foreskin fibroblasts; FBS: foetal bovine serum; SOE: spliced-overlapping-extension; WT: wild type.

\section{Acknowledgements}

The authors would like to thank colleagues from Southern Medical University who provided advice, helped with experiments and provided valuable support.

\section{Funding}

This study was supported by grants from the National Natural Science Foundation of China $(31572506,30972578$ ) and the Science and Technology Planning Project of Guangdong Province, China (2013B060300031).

Availability of data and materials

All data generated or analysed during this study are included in this published article and its additional files.

\section{Authors' contributions}

All authors contributed significantly to this study. HC, YG, PY and KW conceived the study and coordinated its implementation. $H C, Y G, Y Q, X G$ and $\mathrm{KW}$ participated in the experimental design. $\mathrm{HC}, \mathrm{YG}, \mathrm{YQ}, \mathrm{HH}, \mathrm{CL}, \mathrm{ML}$ and $\mathrm{PY}$ performed the experiments and analysed the results. $\mathrm{HC}$ and $\mathrm{YG}$ wrote and revised the manuscript, and $Y Q, M L$ and $K W$ critically revised the manuscript. All authors read and approved the final manuscript.

\section{Ethics approval and consent to participate}

All animal experiments were conducted according to the guidelines established by the Association for Assessment and Acceleration of Laboratory Animal Care International. Animal experimental procedures were approved by the Nanfang Hospital Animal Ethic Committee (permit number: NFYY2017-99), and animal care was performed in accordance with institutional guidelines.

\section{Consent for publication}

Not applicable. 


\section{Competing interests}

The authors declare that they have no competing interests.

\section{Publisher's Note}

Springer Nature remains neutral with regard to jurisdictional claims in published maps and institutional affiliations.

Received: 17 September 2018 Accepted: 5 March 2019

Published online: 15 March 2019

\section{References}

1. Dubey JP. Toxoplasmosis of animals and humans. 2nd ed. Boca Raton: CRC Press; 2010.

2. Zhou DH, Yuan ZG, Zhao FR, Li HL, Zhou Y, Lin RQ, et al. Modulation of mouse macrophage proteome induced by Toxoplasma gondii tachyzoites in vivo. Parasitol Res. 2011:109:1637-46.

3. Puvanesuaran VR, Noordin R, Balakrishnan V. Genotyping of Toxoplasma gondii isolates from wild boars in Peninsular Malaysia. PLoS One. 2013;8:e61730.

4. Leveque MF, Berry L, Besteiro S. An evolutionarily conserved SSNA1/DIP13 homologue is a component of both basal and apical complexes of Toxoplasma gondii. Sci Rep. 2016:6:27809.

5. Dubey JP. Advances in the life cycle of Toxoplasma gondii. Int J Parasitol. 1998;28:1019-24.

6. Guenter W, Bielinski M, Deptula A, Zalas-Wiecek P, Piskunowicz M, Szwed $\mathrm{K}$, et al. Does Toxoplasma gondii infection affect cognitive function? A case control study. Folia Parasitol (Praha). 2012:59:93-8.

7. Goodwin D, Hrubec TC, Klein BG, Strobl JS, Werre SR, Han Q, et al. Congenital infection of mice with Toxoplasma gondii induces minimal change in behavior and no change in neurotransmitter concentrations. J Parasitol. 2012;98:706-12.

8. Stock AK, von Heinegg EH, Kohling HL, Beste C. Latent Toxoplasma gondii infection leads to improved action control. Brain Behav Immun. 2014;37:103-8.

9. Gale SD, Brown BL, Erickson LD, Berrett A, Hedges DW. Association between latent toxoplasmosis and cognition in adults: a cross-sectional study. Parasitology. 2015;142:557-65.

10. Berdoy M, Webster JP, Macdonald DW. Fatal attraction in rats infected with Toxoplasma gondii. Proc Biol Sci. 2000;267:1591-4.

11. Fekadu A, Shibre T, Cleare AJ. Toxoplasmosis as a cause for behaviour disorders - overview of evidence and mechanisms. Folia Parasitol (Praha) 2010;57:105-13.

12. da Silva RC, Langoni H. Toxoplasma gondii: host-parasite interaction and behavior manipulation. Parasitol Res. 2009:105:893-8.

13. Prandovszky E, Gaskell E, Martin H, Dubey JP, Webster JP, McConkey GA. The neurotropic parasite Toxoplasma gondii increases dopamine metabolism. PLoS One. 2011;6:e23866.

14. Arbune AA, Arbune M, Stefanescu V. Parkinsonian syndrome and toxoplasmic encephalitis. J Crit Care Med (Targu Mures). 2016;2:89-92.

15. Webster JP, McConkey GA. Toxoplasma gondii-altered host behaviour: clues as to mechanism of action. Folia Parasitol (Praha). 2010:57:95-104.

16. Gaskell EA, Smith JE, Pinney JW, Westhead DR, McConkey GA. A unique dual activity amino acid hydroxylase in Toxoplasma gondii. PLoS One. 2009;4:e4801

17. Chao HM, Richardson MA. Aromatic amino acid hydroxylase genes and schizophrenia. Am J Med Genet. 2002;114:626-30.

18. Khattak AM, Khan J, Rahman H. Seropositivity of Toxoplasma gondii latent infection in patients with neuropsychiatric disorders. Khyber Med Univ J. 2015;7:14.

19. Elsheikha HM, Busselberg D, Zhu XQ. The known and missing links between Toxoplasma gondii and schizophrenia. Metab Brain Dis. 2016;31:749-59.

20. Maeder ML, Thibodeau-Beganny S, Osiak A, Wright DA, Anthony RM, Eichtinger $M$, et al. Rapid "open-source" engineering of customized zinc-finger nucleases for highly efficient gene modification. Mol Cell. 2008;31:294-301.

21. Christian M, Cermak T, Doyle EL, Schmidt C, Zhang F, Hummel A, et al. Targeting DNA double-strand breaks with TAL effector nucleases. Genetics. 2010;186:757-61.
22. Miller JC, Tan S, Qiao G, Barlow KA, Wang J, Xia DF, et al. A TALE nuclease architecture for efficient genome editing. Nat Biotechnol. 2011;29:143-8.

23. Boch J, Scholze H, Schornack S, Landgraf A, Hahn S, Kay S, et al. Breaking the code of DNA binding specificity of TAL-type III effectors. Science. 2009:326:1509-12.

24. Moscou MJ, Bogdanove AJ. A simple cipher governs DNA recognition by TAL effectors. Science. 2009:326:1501.

25. Ousterout DG, Gersbach CA. The development of TALE nucleases for biotechnology. Methods Mol Biol. 2016:1338:27-42.

26. Sanjana NE, Cong L, Zhou Y, Cunniff MM, Feng G, Zhang F. A transcription activator-like effector toolbox for genome engineering. Nat Protoc. 2012;7:171-92.

27. Cong L, Zhou R, Kuo YC, Cunniff M, Zhang F. Comprehensive interrogation of natural TALE DNA-binding modules and transcriptional repressor domains. Nat Commun. 2012;3:968.

28. Bibikova M, Beumer K, Trautman JK, Carroll D. Enhancing gene targeting with designed zinc finger nucleases. Science. 2003;300:764

29. Shen B, Brown KM, Lee TD, Sibley LD. Efficient gene disruption in diverse strains of Toxoplasma gondii using CRISPR/CAS9. MBio. 2014;5:e01114-214.

30. Sidik SM, Hackett CG, Tran F, Westwood NJ, Lourido S. Efficient genome engineering of Toxoplasma gondii using CRISPR/Cas9. PLoS One. 2014;9:e100450

31. Zhao G, Pu J, Tang B. Applications of ZFN, TALEN and CRISPR/Cas9 techniques in disease modeling and gene therapy. Zhonghua Yi Xue Yi Chuan Xue Za Zhi. 2016:33:857-62 (In Chinese).

32. Ni PL, Liu C, Chen LY. DNA Scissors-TALEN and CRISPR/Cas. Chin J Cell Biol. 2014:1:5-11.

33. Cheng SQ, Wang ZR. Establishment of pregnant mice infection model with prugniaud strain of Toxoplasma gondii. J Guiyang Med Coll. 2015;3:257-9 (In (hinese)

34. Wang ZT, Harmon S, O'Malley KL, Sibley LD. Reassessment of the role of aromatic amino acid hydroxylases and the effect of infection by Toxoplasma gondii on host dopamine. Infect Immun. 2015;83:1039-47.

35. Marino ND, Boothroyd JC. Toxoplasma growth in vitro is dependent on exogenous tyrosine and is independent of $\mathrm{AAH} 2$ even in tyrosine-limiting conditions. Exp Parasitol. 2017:176:52-8.

36. Afonso C, Paixao VB, Klaus A, Lunghi M, Piro F, Emiliani C, et al. Toxoplasmainduced changes in host risk behaviour are independent of parasite-derived AaaH2 tyrosine hydroxylase. Sci Rep. 2017;7:13822.

37. McFarland R, Wang ZT, Jouroukhin Y, Li Y, Mychko O, Coppens I, et al. AAH2 gene is not required for dopamine-dependent neurochemical and behavioral abnormalities produced by Toxoplasma infection in mouse. Behav Brain Res. 2018;347:193-200.

38. Soldati D, Boothroyd JC. A selector of transcription initiation in the protozoan parasite Toxoplasma gondii. Mol Cell Biol. 1995;15:87-93.

39. Kim K, Soldati D, Boothroyd JC. Gene replacement in Toxoplasma gondi with chloramphenicol acetyltransferase as selectable marker. Science. 1993;262:911-4.

40. Han SQ, Wu K. Advances in the study of bradyzoite-associated proteins of Toxoplasma gondii. Chin J Zoonoses. 2014;9:965-70.

41. Burg JL, Perelman D, Kasper LH, Ware PL, Boothroyd JC. Molecular analysis of the gene encoding the major surface antigen of Toxoplasma gondii. J Immunol. 1988;141:3584-91.

42. An N, Zhang R. Cloning and identification of promoter of special expression protein SAG1 of Toxoplasma gondii tachyzoite. Chin J Biologicals. 2010;23:602-4.

43. Nishikawa $Y$, Xuenan $X$, Makala L, Vielemeyer $O$, Joiner KA, Nagasawa $H$. Characterisation of Toxoplasma gondii engineered to express mouse interferon-gamma. Int J Parasitol. 2003;33:1525-35.

44. van den Hoff MJ, Moorman AF, Lamers WH. Electroporation in "intracellular" buffer increases cell survival. Nucleic Acids Res. 1992;20:2902.

45. Zhang $\mathrm{H}$, Zhang $\mathrm{Y}$, Cao J, Zhou Y, Wang N, Zhou J. Determination of stage interconversion in vitro and in vivo by construction of transgenic Toxoplasma gondii that stably express stage-specific fluorescent proteins. Exp Parasitol. 2013;134:275-80

46. Striepen $B, H e C Y$, Matrajt M, Soldati D, Roos DS. Expression, selection, and organellar targeting of the green fluorescent protein in Toxoplasma gondii. Mol Biochem Parasitol. 1998;92:325-38. 